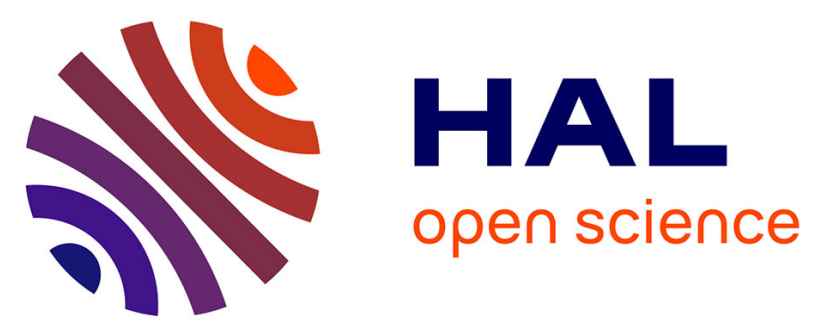

\title{
Reliable estimation of the kinetic parameters of redox enzymes by taking into account mass transport towards rotating electrodes in protein film voltammetry experiments
}

Meriem Merrouch, Jessica Hadj-Saïd, Christophe Léger, Sebastien Dementin, Vincent Fourmond

\section{To cite this version:}

Meriem Merrouch, Jessica Hadj-Saïd, Christophe Léger, Sebastien Dementin, Vincent Fourmond. Reliable estimation of the kinetic parameters of redox enzymes by taking into account mass transport towards rotating electrodes in protein film voltammetry experiments. Electrochimica Acta, 2017, 245, pp.1059-1064. 10.1016/j.electacta.2017.03.114 . hal-01614234

\section{HAL Id: hal-01614234 \\ https://hal-amu.archives-ouvertes.fr/hal-01614234}

Submitted on 16 Apr 2018

HAL is a multi-disciplinary open access archive for the deposit and dissemination of scientific research documents, whether they are published or not. The documents may come from teaching and research institutions in France or abroad, or from public or private research centers.
L'archive ouverte pluridisciplinaire HAL, est destinée au dépôt et à la diffusion de documents scientifiques de niveau recherche, publiés ou non, émanant des établissements d'enseignement et de recherche français ou étrangers, des laboratoires publics ou privés. 


\title{
Reliable estimation of the kinetic parameters of redox enzymes by taking into account mass transport towards rotating electrodes in protein film voltammetry experiments
}

\author{
Meriem Merrouch ${ }^{\mathrm{a}}$, Jessica Hadj-Saïd ${ }^{\mathrm{a}}$, Christophe Léger $^{\mathrm{a}}$, Sébastien Dementin ${ }^{\mathrm{a}}$, Vincent Fourmond ${ }^{* a}$ \\ ${ }^{a}$ Aix-Marseille Université, CNRS, BIP UMR 7281, 31 chemin J. Aiguier, F-13402 Marseille cedex 20, France
}

\begin{abstract}
In Protein Film Voltammetry, a redox enzyme is immobilized on a rotating electrode in a configuration allowing fast, direct electron transfer. This technique is used to probe the mechanism of enzymes by quantitatively interpreting the response in current as a function of the experimental conditions. Limitation by mass transport of the substrate towards the electrode may obscure important features and complicate the analysis of the enzymatic response, so much so that the enzyme has high activity. In this work, we derive equations taking into account mass transport of substrate, for the steady-state current generated by an enzyme following Michaelis-Menten kinetics and immobilized onto a hydrodynamic (e.g. rotating) electrode. We use these equations to model the current response of films of CO-dehydrogenase, a metalloenzyme that catalyzes the oxidation of $\mathrm{CO}$, to transient exposures to its gaseous substrate. We show that neglecting transport yields poor fits and overestimated, unreliable, values of $K_{m}$ (even when using the Koutecky-Levich approximation), whereas taking into account transport yields much better fits and more reliable parameters. We reinterpret previously published data by taking into account transport limitations.
\end{abstract}

Keywords: protein film voltammetry; mass transport; CO dehydrogenase; Koutecky-Levich; gaseous substrates

\section{Introduction}

Protein Film Voltammetry (PFV) has been used for two decades to study the mechanism of redox enzymes[1-3]. In this technique, an enzyme is immobilized onto a rotating electrode in such a way that electron transfer is direct and fast. This technique was used to study catalytic signals, i.e. signals resulting from the catalytic oxidation or reduction of the enzyme's substrate at the electrode. The resulting catalytic current, which is proportional to the enzymatic activity, lends itself well to quantitative interpretation: one can monitor its evolution over time to learn about inactivation/activation processes[4-7], or model its dependence on electrode potential to learn about the steps in the catalytic cycle[8-10]. In catalytic PFV, the substrate is consumed at the electrode by the catalytic reaction. To avoid depletion, electrochemists have therefore used rotating disc electrodes, taking care that the electrode is rotated fast enough that the enzymatic response does not depend on the rotation rate, and hence reflects only the properties of the enzyme. Early reports of the oxidation of $\mathrm{H}_{2}$ at an electrode modified with an hydrogenase showed a strong dependence of the current on the electrode rotation rate, which was modelled using the KouteckyLevich equation[11]. Heering and coworkers[12], Reda and Hirst[13], and Léger and Bertrand[2] derived exact expressions for the current of an enzyme following Michaelis-Menten kinetics taking into account mass transport.

$\mathrm{CO}$ dehydrogenases are enzymes that employ a $\mathrm{NiFe}_{4} \mathrm{~S}_{4}$ active site to catalyze the reversible oxidation of $\mathrm{CO}$ to $\mathrm{CO}_{2}[14]$.

\footnotetext{
*To whom correspondance should be addressed. Email address: vincent.fourmond@imm.cnrs.fr, Phone: +33491164536
}

They are extremely fast[15], with turnover rates up to $4 \times 10^{4} \mathrm{~s}^{-1}$, which makes them desirable for use in biotechnological devices, where they could be combined for instance with hydrogenases to catalyse the water-gas shift reaction[16]. Distinct CODHs have been studied using PFV in the past decade, such as CODH I and II from Carboxydothermus hydrogenoformans or CODH from Desulfovibrio vulgaris[17-20]. However, the high activity of CODHs introduces a complication for their study using PFV, since many of the electrochemical signals we recorded with Desulfovibrio vulgaris CODH adsorbed onto pyrolytic graphite edge electrodes showed signs of limitation by transport, even when rotating the electrode at $6 \mathrm{krpm}$; we thus decided to include transport limitation in our modelling.

In this work, we derive equations slightly different from those of the previous works[13, 2] for the current given by a redox enzyme following Michaelis-Menten kinetics and immobilized on an electrode, taking into account transport limitation. We use this model to interpret the response of films of Desulfovibrio vulgaris $\mathrm{CODH}$ to transient exposures to $\mathrm{CO}$, and reinterpret data previously published by others[17]. We show that neglecting mass transport leads to unreliable, overestimated values of the $K_{m}$ parameter. In addition, we show that using the Koutecky-Levich analysis does not improve the reliability of the determined $K_{m}$ values, but yields to largely underestimated values. Finally, we propose a simple method to model the data.

\section{Formalism}

In this paper, we consider the enzymatic oxidation of a substrate at a hydrodynamic electrode. We assume that the hydrodynamic flow towards the electrode imposes a flux of substrate 
that takes the following form[21, 22]:

$$
J_{\mathrm{t}}=m\left(c^{\mathrm{b}}-c^{0}\right)
$$

where $J_{\mathrm{t}}$ is the flux of substrate towards the electrode, $c^{\mathrm{b}}$ is the concentration of substrate in the "bulk" (i.e. far away from the electrode), $c^{0}$ is the concentration at the electrode surface, and $m$ is the mass-transfer coefficient[21], which depends on the nature of the flow and on its velocity.

At the electrode, we consider that the substrate is oxidized by an enzymatic reaction with a rate per molecule of enzyme given by:

$$
k=\frac{k_{\mathrm{cat}}}{1+\frac{K_{m}}{c^{0}}}=\frac{k_{\mathrm{eff}} c^{0}}{1+\frac{c^{0}}{K_{m}}}
$$

where $k_{\text {cat }}$ the maximum catalytic rate, $K_{m}$ the Michaelis constant for the oxidation of the substrate, and $k_{\text {eff }}$ the catalytic efficiency, defined as:

$$
k_{\mathrm{eff}}=\frac{k_{\mathrm{cat}}}{K_{m}}
$$

The electrode, covered with the enzyme with a surface concentration $\Gamma$, consumes a flux of substrate given by:

$$
J_{\mathrm{c}}=\Gamma \frac{k_{\mathrm{eff}} c^{0}}{1+\frac{c^{0}}{K_{m}}}
$$

We now consider three models that differ in the way the transport is taken into account.

In model (a), the transport is assumed to be so fast that $c^{0}$ is always equal to $c^{\mathrm{b}}$. In that case, the electrical current density is given by:

$$
j_{(\mathbf{a})}=n F J=n F \Gamma \frac{k_{\mathrm{eff}} c^{\mathrm{b}}}{1+c^{\mathrm{b}} / K_{m}}=n F \Gamma \frac{k_{\mathrm{cat}}}{1+K_{m} / c^{\mathrm{b}}}
$$

in which $n$ is the number of electrons involved in the oxidation reaction, and $F$ the Faraday constant.

In model (b), we assume that the transport can be taken into account using the Koutecky-Levich approximation, so that:

$$
1 / j_{(\mathbf{b})}=1 / j_{\text {Levich }}+1 / j_{\text {enzyme }}
$$

in which $j_{\text {Levich }}$ is the so-called Levich current, completely limited by mass-transport (obtained for $c^{0}=0$ ) and $j_{\text {enzyme }}=j_{(\mathbf{a})}$ is the current given by the system when the transport is infinitely fast (equation (5)). This gives:

$$
\begin{aligned}
j_{(\mathbf{b})} & =n F \Gamma \frac{k_{\mathrm{eff}}^{\mathrm{app}} c^{\mathrm{b}}}{1+c^{\mathrm{b}} / K_{m}^{\mathrm{app}}} \\
k_{\mathrm{eff}}^{\mathrm{app}} & =\frac{k_{\mathrm{eff}}}{1+\mu / m} \\
K_{m}^{\mathrm{app}} & =K_{m}(1+\mu / m)
\end{aligned}
$$

where we define $\mu=\Gamma k_{\text {eff. }}$ Model (b) is therefore formally equivalent to model (a), with only a different meaning of the values of $K_{m}$ and $k_{\text {eff. }}$.

In the last model, model (c), we make no hypothesis. Instead we determine the concentration $c^{0}$ of substrate at the electrode using the conservation of mass. This model gives therefore the true value of the current, unlike models (a) and (b) which are approximations. Under steady state conditions, $J_{\mathrm{c}}=$ $J_{\mathrm{t}}$, which gives the following equation for $c^{0}$ :

$$
m\left(c^{\mathrm{b}}-c^{0}\right)=\Gamma \frac{k_{\mathrm{eff}} c^{0}}{1+\frac{c^{0}}{K_{m}}}=\frac{\mu}{1+\frac{c^{0}}{K_{m}}} c^{0}
$$

Computing $c^{0}$ and substituting into either (1) or (4) yields the following expression for the electrical current density:

$$
\begin{aligned}
j_{(\mathbf{c})} & =n F \Gamma \frac{k_{\mathrm{eff}} K_{m}}{1+\frac{K_{m}}{c_{0}}} \\
c^{0} & =c^{\mathrm{b}} \frac{\gamma+\sqrt{\gamma^{2}+4 \frac{K_{m}}{c^{\mathrm{b}}}}}{2} \\
\gamma & =1-\frac{K_{m}}{c^{\mathrm{b}}}\left(1+\frac{\mu}{m}\right)
\end{aligned}
$$

Similar expressions, using $k_{\text {cat }}$ rather than $k_{\text {eff }}$, have been derived before[12, 13, 2]. Expression (9) has the advantage of making it clear which non-dimensional parameters influence the current: $c^{\mathrm{b}} / K_{m}$ and $\mu / m$.

Below, we compare the dependence of the current on the normalized concentration $c^{\mathrm{b}} / K_{m}$ for models (b) and (c), and we study how the current is affected by the value of $\mu / m$, which quantifies the competition between enzymatic substrate oxidation and transport. If $\mu / m$ is greater than 1 , then catalysis is faster than transport, and vice-versa for values lower than 1. For $\mu / m=0$, both model (b) and model (c) fall back to model (a), which does not take transport into account. Under these conditions, the dependence of the current on the bulk concentration truly reflects the properties of the enzyme.

When $c^{\mathrm{b}}$ tends to infinity, regardless of the model used, the current density reaches a maximum value $j_{\max }$ :

$$
j_{\max }=n F \Gamma k_{\mathrm{eff}} K_{m}
$$

We used this value to normalize the current density for all models.

Figure 1a shows curves of $j / j_{\max }$ against $c^{\mathrm{b}} / K_{m}$ for several values of $\mu / m$ for model (b) (equation (7), dotted lines) and for model (c) (equation (9), solid lines). All curves show a similar behaviour, with a linear region at low concentration and a saturation at high concentration. The current is not significantly affected by the limitation by transport up to values of $\mu / m$ around 0.3 , but it deviates significantly for higher values of $\mu / m$. Increasing $\mu / m$ increases the concentration at which saturation is reached, and decreases the slope of the initial linear response. Regarding the comparison between models (b) and (c), figure 1a shows that, apart from the initial linear region where both models give the same values, the Koutecky-Levich model (model (b)) systematically underestimates the current with respect to the exact model (model (c)).

The current in the initial linear region can be computed by considering the limit $c^{\mathrm{b}} \rightarrow 0$ in equation (9) (model (c)), which gives:

$$
j=n F \frac{\Gamma k_{\mathrm{eff}} c^{\mathrm{b}}}{1+\mu / m}
$$



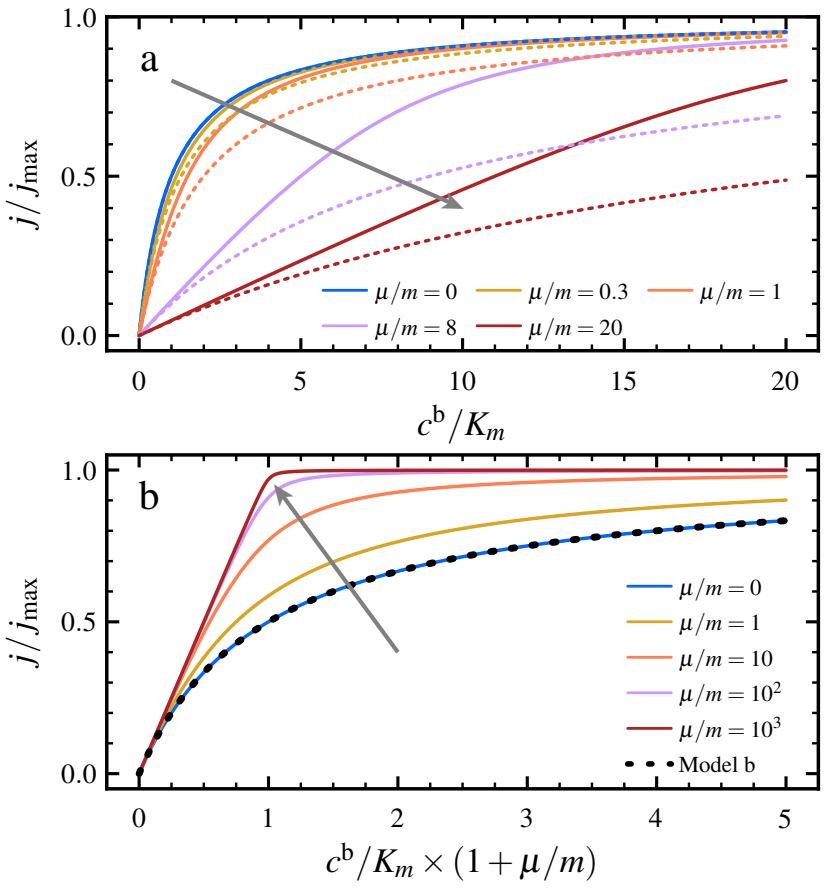

Figure 1: Panel a: normalized current density of model (b) (dotted lines) or model (c) (solid lines) as a function of either the normalized concentration $c_{\mathrm{b}} / K_{m}$ for different values of the $\mu / m$ ratio. Panel b: normalized values of the current density of model (c) as a function $c_{\mathrm{b}} / K_{m} \times(1+\mu / m)$ for different values of $\mu / m$. In this representation, model (b) gives a curve independent of $\mu / m$, drawn as thick dark dots. In both panels, the arrow points in the direction of increasing $\mu / m$.

Model (b) also gives the same expression for the current in the linear region.

In figure 1a, we have plotted the normalized current for only a small range of values of $\mu / m$, since increasing this value yields curves with very low initial slopes and saturations at too high concentrations. Equation (11) suggests that plotting the current as a function of $(1+\mu / m) \times c^{\mathrm{b}} / K_{m}$ should make it easier to compare curves with very different values of $\mu / m$, as the initial linear regions should all match, as should the plateaus at $j / j_{\max }=1$. We have done so in figure $1 \mathrm{~b}$. In this representation, the curves given by model (b) do not depend on $\mu / m$ anymore, and match that of model (c) at $\mu / m=0$. Figure $1 \mathrm{~b}$ shows that as the limitation by the transport becomes more important (the $\mu / m$ ratio increases), the transition between the initial linear region and the plateau current becomes more abrupt, until, at high values of $\mu / m$, the graph becomes two lines that intersect at $(1+\mu / m) \times c^{\mathrm{b}} / K_{m}=1$. This sharp transition is reproduced neither by model (a), that does not take transport into account, nor by model (b), for which the modelling of transport is too crude; this sharp transition is characteristic of transport limitations.

\section{Experimental}

\section{Protein purification}

Desulfovibrio vulgaris $\mathrm{CO}$ dehydrogenase was purified as before[19].

\section{Electrochemistry}

Electrochemical experiments were conducted as before[20].

\section{Data modelling}

To model experimental data with equation (9), it is more convenient to model the current directly rather than the current density, which is just the current multiplied by $A$ the electrode area. We used QSoas for data fitting, using the following Ruby function:

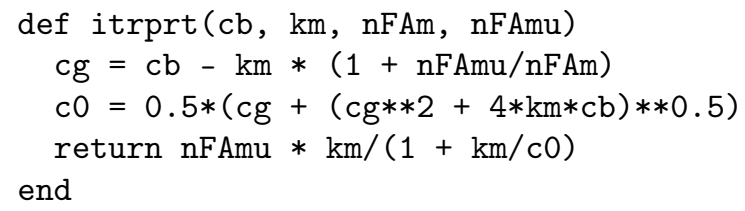

To use this function, we saved it as a function.rb text file and loaded it using the command:

ruby-run function.rb

The fits were run using the following command:

fit-arb itrprt (s, km,nFAm, nFAmu)+io /with=s:1, exp

The /with=s:1, exp specifies that the parameter $\mathrm{s}$ varies according to equation (12). $\mathrm{km}$ is the $K_{m}$ parameter, $\mathrm{nFAm}$ is the value of $n F A m$ and $n F A m u$ that of $n F A \mu$ (it is not possible to determine $m$ or $\mu$ from the fit without knowning $A$ ). We use +io to account for a slight offset with respect to $I=0$. Fitting equation (5) to data can be achieved using the following command:

fit-arb nFAmu*s/(km+s)+io/with=s: $1, \exp$

\section{Results}

CO dehydrogenase from Desulfovibrio vulgaris forms stable and very active films on pyrolytic graphite edge electrodes[20]. Using a rotating disc electrode configuration, we have submitted such films to injections of aliquots of $\mathrm{CO}$ in the electrochemical buffer. Upon injection, the concentration of $\mathrm{CO}$ in the bulk increases instantly, and then the exchange between the electrochemical buffer and the atmosphere, facilitated by the electrode rotation, results in an exponential decrease of the concentration over time[23, 24]:

$$
c^{\mathrm{b}}= \begin{cases}c^{\mathrm{inj}} \exp \left(-\frac{t-t_{0}}{\tau}\right) & \text { if } t>t_{0}, \\ 0 & \text { otherwise }\end{cases}
$$

where $c^{\text {inj }}$ is the injected concentration, $t_{0}$ the time of the injection and $\tau$ the time constant of the decrease of $\mathrm{CO}$. This makes it possible to probe the response of $\mathrm{CODH}$ to a large range of concentrations of $\mathrm{CO}$ in a single experiment.

Figure 2 shows the current monitored at a fixed potential produced by an electrode modified with a film of $\mathrm{CODH}$ submitted to a single injection of an aliquot of $\mathrm{CO}$. Initially, there is no substrate, and therefore no current. The injection at $t=t_{0}=0$ results in the appearance of a $\mathrm{CO}$ oxidation current that has 

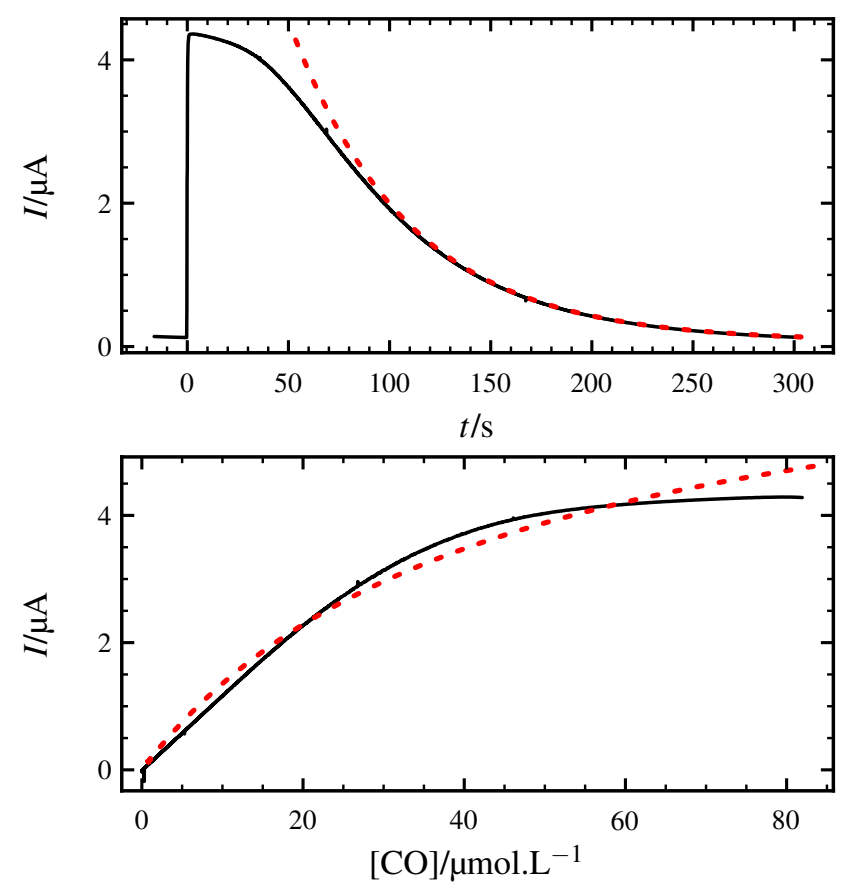

Figure 2: Panel a: current generated by a film of $D v$ CODH submitted to a single injection of $83 \mu \mathrm{M} \mathrm{CO}$ at $t=0$. The red trace is the exponential fit to the tail of the current $(\tau=60.8 \pm 0.3 \mathrm{~s})$. Panel $\mathrm{b}$ : current as a function of the concentration of $\mathrm{CO}$ deduced from the time constant of the fit in panel a. The red trace is a fit of model (a) (equation (5)). Conditions: $T=25^{\circ} \mathrm{C}, \mathrm{pH}$, $\omega=4 \mathrm{krpm}$, electrode potential: $-310 \mathrm{mV}$.

a vaguely sigmoidal shape, with an initial plateau, an inflexion point, and a perfect mono-exponential tail. The latter mirrors the exponential decrease of the concentration of $\mathrm{CO}$, because, as the concentration decreases, it reaches low enough values that the current becomes proportional to the concentration (equation (11)). Therefore, fitting a mono-exponential decay to the the tail of the current yields the time constant $\tau$ of the decay of the bulk concentration of CO. This makes it possible to determine the whole evolution of the concentration of CO over time, using equation (12), because both $c^{\text {inj }}$ and $t_{0}$ are known.

We have taken advantage of this possibility to plot the current as a function of the bulk concentration of $\mathrm{CO}$ in figure $2 \mathrm{~b}$. While the $I=f([\mathrm{CO}])$ curve follows a saturation behaviour, with a large region in which the current depends linearly on the concentration before reaching a plateau at higher concentrations, the transition between the linear region and the plateau is more abrupt than that of model (a), which neglects transport limitation. This is attested by the poor fit of model (a) (equation (5)) to the data (red dashed curve in figure $2 b$ ). In fact, the experimental $I=f([\mathrm{CO}])$ trace of figure $2 \mathrm{~b}$ is much closer in appearance to the $\mu / m=8$ model (c) trace of figure 1a.

To determine the $K_{m}$ of the $\mathrm{CODH}$ under the experimental conditions used, there are two strategies: (i) one can fit model (c) (equation (9)) to the dependence of the current on the bulk concentration of CO (figure 2b), which was itself deduced by extrapolating the exponential decrease fitted in the tail of the current. (ii) alternatively, one can directly substitute

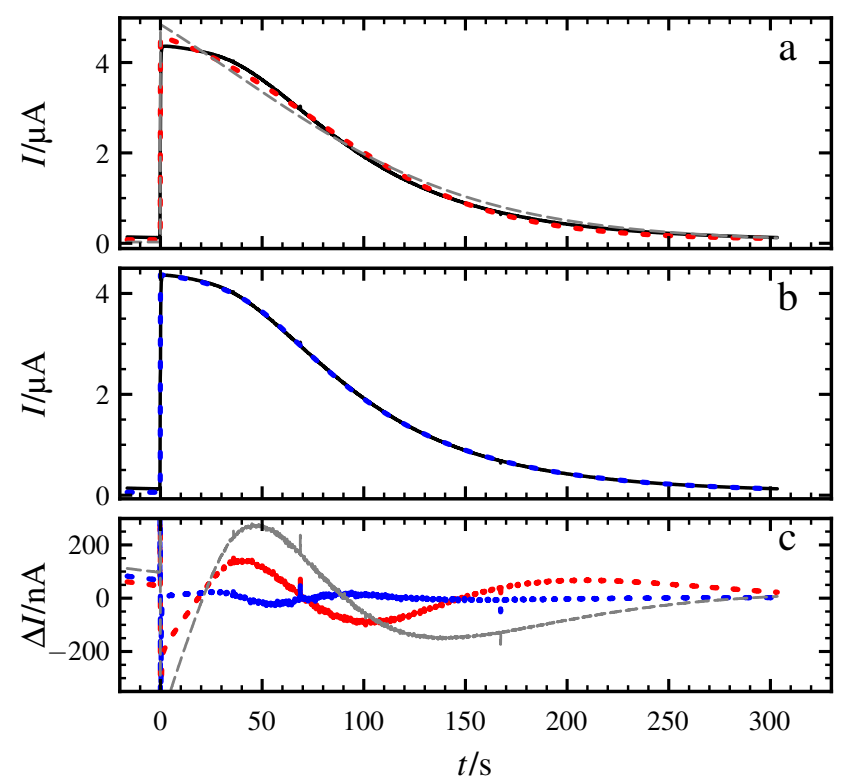

Figure 3: Panels a \& b, black trace: same data as in figure 2a. Panel a, red dashed trace: fit of equation (5) (model (a)) with $c^{\mathrm{b}}$ given by (12) to the data; dashed gray trace: fit of the same model, but with the value of $\tau$ fixed to that determined by the exponential fit in figure 2a. Panel b, blue dashed trace: fit of equation (9) (model (c)) with $c^{\mathrm{b}}$ given by (12) to the data. Panel c: residuals of the fit, with the same color code as in panels a\&b. Conditions as in figure 2 . Parameters of the fit $\left(A=4.15 \mathrm{~mm}^{2}\right.$ is the area of the electrode), panel a, red trace: $K_{m}=13.7 \pm 0.3 \mu \mathrm{M}, n F A \Gamma k_{\mathrm{eff}}=380 \pm 70 \mathrm{nA} . \mu \mathrm{M}^{-1}$ (corresponding to $\left.I_{\max }=5.24 \pm 0.03 \mu \mathrm{A}\right), \tau=42.7 \pm 0.3 \mathrm{~s}$; gray trace: $K_{m}=45 \pm 1 \mu \mathrm{M}$, $n F A \Gamma k_{\text {eff }}=164 \pm 1 \mathrm{nA} . \mu \mathrm{M}^{-1}$ (corresponding to $\left.I_{\max }=7.4 \pm 0.3 \mu \mathrm{A}\right), \tau$ fixed to $60.8 \mathrm{~s}$. Panel b: $K_{m}=2.2 \pm 0.1 \mu \mathrm{M}, n F A \Gamma k_{\mathrm{eff}}=2.1 \pm 0.1 \mu \mathrm{A} . \mu \mathrm{M}^{-1}$ (corresponding to $I_{\max }=4.50 \pm 0.01 \mu \mathrm{A}$ ), $n F A m=127 \pm 2 \mathrm{nA} \mu \mathrm{M}^{-1}, \tau=$ $60.8 \pm 0.3 \mathrm{~s}$.

equation (12) into equation (9) and fit the resulting expression to the raw data. As the equation for model (b) is strictly equivalent to that of model (a), fitting with model (a) in fact gives the values of $K_{m}$ for both models, provided $\mu / m$ is determined independently.

We chose option (ii), since it requires only one fit, and therefore is less prone to systematic errors. Moreover, this is also how we proceeded before[23] (but using model (a)). We also followed the same strategy but using model (a) (equation (5)) to the data to assess the extent to which neglecting completely transport affects the quality of the fits and of the determined parameters.

Figure 3a shows a fit of model (a) to the data plotted in figure $2 \mathrm{a}$. The fit is not very good, much more symmetric around the inflexion point than the data really are. Moreover, the exponential decrease is not well reproduced, and the fitted model decreases faster than the real current, which means that the extrapolated concentration of $\mathrm{CO}$ is not correct. Imposing the correct value of $\tau$, as determined from the fit in figure 2 yielded even poorer fits (gray lines in figure $3 \mathrm{a}$ and $\mathrm{c}$ ). On the other hand, fitting model (c) to the data (figure $3 b$ ) yields a perfect fit that reproduces both the exponential decrease and the noticeable asymmetry around the inflexion point (the value of $\tau=60.8 \mathrm{~s}$ determined from this fit is the same as that determined directly using a simple mono-exponential decay). The 


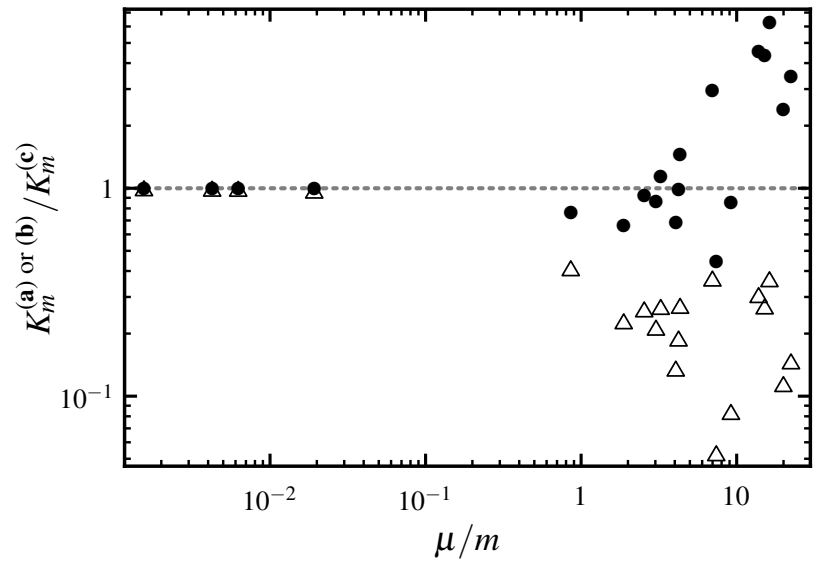

Figure 4: Systematic fits of models (a) and (c) to responses to injections of $\mathrm{CO}$ of $D v \mathrm{CODH}$ films. Full circles: values of $K_{m}^{(\mathbf{a})} / K_{m}^{(\mathbf{c})}$ as a function of $\mu / m$ (determined from fits of model (c)). Open triangles: values of $K_{m}^{(\mathbf{b})} / K_{m}^{(\mathbf{c})}$ as a function of $\mu / m$; the value of $K_{m}^{(\mathbf{b})}$ is determined from equation (7c) and the value of $\mu / m$ determined from fits of model (c). Conditions variable, $\mathrm{pH} 7$, $25^{\circ} \mathrm{C}$, electrode potential from $-460 \mathrm{mV}$ to $-410 \mathrm{mV}$.

better quality of the fit ${ }^{1}$ the full model is confirmed by the inspection of the residuals in figure $3 \mathrm{c}$, since those for model (c) (in blue) are much smaller and evenly distributed around zero than those of model (a) (in red). From the fitted value of $n$ F A m ( $A$ is the electrode area, $A=0.0415 \mathrm{~cm}^{2}$ ), one can compute a value of the mass transport coefficient of $m=16 \times 10^{-3} \mathrm{~cm} . \mathrm{s}^{-1}$. For a rotating disc electrode, the mass transport coefficient takes the form[21]:

$$
m=0.62 D^{2 / 3} \omega^{1 / 2} v^{-1 / 6}
$$

where $D$ is the diffusion coefficient of the $\mathrm{CO}, \omega$ the angular velocity of the RDE, and $v$ is the kinematic viscosity of the solution. As the the kinematic viscosity of water of $v=$ $10^{-2} \mathrm{~cm}^{2} \cdot \mathrm{s}^{-1}$ and the rotation rate is $4 \mathrm{krpm}$, we obtain a coefficient for the diffusion of $\mathrm{CO}$ in the electrochemical buffer of $D_{\mathrm{CO}}=1.4 \times 10^{-5} \mathrm{~cm}^{2} . \mathrm{s}^{-1}$, well in the expected range for the diffusion of a small molecule in water, and compatible with the value of $D_{\mathrm{CO}}=2 \times 10^{-5} \mathrm{~cm}^{2} . \mathrm{s}^{-1}$ determined at $20^{\circ} \mathrm{C}$ by Wise and Houghton from the measure of the rate of collapse of small bubbles in water[25].

As model (c) includes (a) and the known limitation by transport, the $K_{m}$ values determined via model (c) are necessarily closer to the real values. In the above example, the error in the $K_{m}$ value from fitting model (a) to the data rather than model (c) is large, almost an order of magnitude. This prompted us to study in a systematic fashion the error on $K_{m}$ resulting from wrongly fitting model (a) to chronoamperograms in which the limitation by transport cannot be neglected. We have run a number of experiments like that in figure 2, under various conditions. For each, we have fitted both model (a) and model (c) to the resulting current traces. We have obtained two distinct values of $K_{m}: K_{m}^{(\mathbf{a})}$ and $K_{m}^{(\mathbf{c})}$. Using the values of $\mu / m$ determined from the

\footnotetext{
${ }^{1}$ Based on the assumption of a standard deviation of $60 \mathrm{nA}$ in the current, the $\chi^{2}$ "goodness-of-fit" is 1 for model (c) and $10^{-20}$ for model (a).
}

fit of model (c), we could also compute $K_{m}^{(\mathbf{b})}$, the value of $K_{m}$ deduced from the Koutecky-Levich approximation. Figure 4 shows the ratios $K_{m}^{(\mathbf{a})} / K_{m}^{(\mathbf{c})}$ (dots) and $K_{m}^{(\mathbf{b})} / K_{m}^{(\mathbf{c})}$ (open triangles) as a function of $\mu / m$, collected over a range of experimental conditions. For low $\mu / m$ ratios, all three models are equivalent and give exactly same value for the $K_{m}$, as attested by a ratio of 1. However, for $\mu / m>1$ there is large discrepancy between the "true" values and the values given by models (a) and (b), as attested by the significant deviation from the $y=1$ horizontal line. Using model (a) or (b) tends to overestimate or underestimate respectively the $K_{m}$ values.

\section{Discussion}

The use of very active films of redox enzymes in Protein Film Voltammetry can result in depletion of substrate at the electrode, complicating the analysis of the electrocatalytic current. We have derived an equation for the current given by a redox enzyme that follows Michaelis-Menten kinetics immobilized on a hydrodynamic electrode, under conditions where transport limitation is not neglected (model (c), equation (9)). Our approach differs from the previous ones in that we chose to parametrize the enzymatic activity using the catalytic efficiency $k_{\text {eff }}$ rather than the maximum turnover rate. We show that the parameter that governs substrate depletion is the ratio $\Gamma k_{\text {eff }} / m$. Increasing this ratio makes the dependence of current on bulk concentration continuously change from the true Michaelis-Menten enzymatic response when $\Gamma k_{\text {eff }} / m$ is low to a linear response that abruptly saturates to give a plateau when $\Gamma k_{\text {eff }} / m$ is high (figure $1 \mathrm{~b}$ ). Increasing the electroactive coverage or using more active enzymes amplifies the effects of substrate limitation, while increasing the mass-transport coefficient $m$ (by increasing the rotation rate) decreases the effects of substrate limitation. We note that increasing the $\Gamma k_{\text {eff }} / m$ ratio increases the range in which the electrode responds linearly in current to the concentration of substrate, which may be desirable for the construction of sensors with a large operating range.

We showed that using the Koutecky-Levich approximation (model (b), equation (7)) does not change the shape of the $j=$ $f\left(c^{\mathrm{b}}\right)$ curve with respect to the model not taking transport limitation into account (model (a), equation (5)); it just changes the interpretation of the values of $K_{m}$ and $k_{\text {eff }}$ determined from the fit, while requiring an independent measure of the $\mu / m$ ratio. In particular, the Koutecky-Levich model does not reproduce the sharp transition between the low-concentration linear regime and the saturation typical of mass-transport limited configurations (at high values of $\mu / m$ ).

We have applied this model to the specific case of a gas processing enzyme, CO-dehydrogenase. Injections of aliquots of buffer saturated with the substrate in a RDE electrochemical cell results in an exponential decrease over time of the substrate concentration which may be exploited to probe the enzymatic response over a large range of substrate concentration in a single experiment[23].

We have taken advantage of this possibility to characterize CO oxidation by Desulfovibrio vulgaris CO dehydrogenase. 

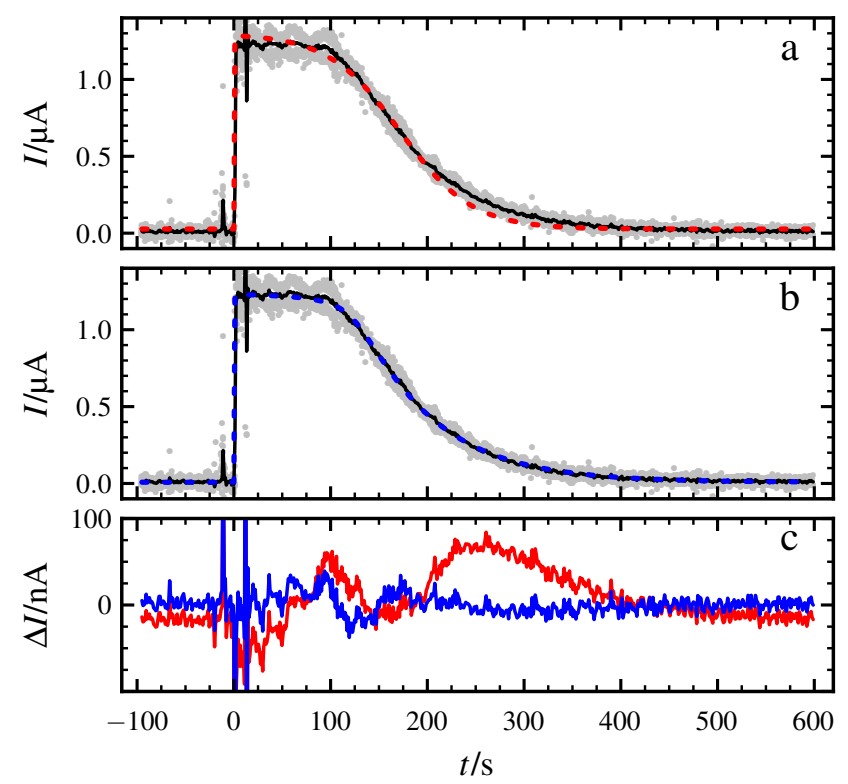

Figure 5: Panels a \& b, gray dots: data from figure S2 of supplementary information of ref. 17, black line: same data, smoothed. Panel a, red dashed trace: original modelling of the data in ref. 17, using model (a). The authors did not fit model (a) directly to the raw data, but rather used a linear fit to $\ln \left(I_{\max } / I-1\right)$. Panel b, blue dashed trace: fit of model (c) to the data. Panel c: residuals of the fit, with the same color code as in panels a\&b. Parameters of the fit ( $A$, the area of the electrode, is unknown, and we assumed from figure S2ii that the injected concentration of CO is $300 \mu \mathrm{M}$ ), panel a: $K_{m}=2.9 \mu \mathrm{M}$, $n F A \Gamma k_{\mathrm{eff}}=428 \mathrm{nA} . \mu \mathrm{M}^{-1}, \tau=39 \mathrm{~s}$. Panel b: $K_{m}=1.6 \pm 0.4 \mu \mathrm{M}$, $n F A \Gamma k_{\mathrm{eff}}=575 \pm 2 \mathrm{nA} \cdot \mu \mathrm{M}^{-1}, n F A m=22 \pm 1 \mathrm{nA} \cdot \mu \mathrm{M}^{-1}, \tau=75 \pm 2 \mathrm{~s}$ Adapted with permission from ref. 17. Copyright 2007 American Chemical Society.

We have shown that fitting model (a) or model (b) to experimental data yields poor fits that misrepresent the exponential decay of the current in the linear part of the response and fail to reproduce the sharp transition between the initial saturation and the subsequent decrease (figure 3a). On the contrary, fitting model (c) (equation (9)) to experimental data yields very good fits with meaningful values of $K_{m}$ and correct values of $m$ (figure $3 \mathrm{~b}$ ). As $m$ is actually determined from the fit, this removes the need for the independent determination of $m$, which would require measuring a diffusion coefficient.

We systematically compared fits of model (a) (neglecting transport) to fits of model (c). We showed that using model (a) yields overestimated and dispersed $K_{m}$ values when the $\mu / m$ parameter is above 1, whereas the fits of model (c) yielded much more reproducible parameter values. Fitting model (b) to the data does not improve reliability, since models (a) and model (b) are strictly equivalent. However, the determination of $K_{m}$ values from fits of model (b) requires independent determination of the value of $m$, and yields greatly underestimated values.

Parkin and coworkers have used model (a) to analyse experiments similar to that in figure 2, to determine the value of $K_{m}$ for Carboxydothermus hydrogenoformans CODH I[17]. Figure 5 shows the response in current of a film of $C$. hydrogenoformans $\mathrm{CODH} \mathrm{I}$ to an injection of an aliquot of CO-saturated buffer (adapted from ref. 17, figure S2). The resulting current is very similar to the data shown in figure $2 \mathrm{a}$, including the asymmetry around the inflexion point that is indicative of mass-transport limitation.

We have reproduced the original data modelling using (a) in figure 5a, and we have fitted model (c) to the data in figure $5 \mathrm{~b}$. As can be seen from the residuals (figure 5c), the fit of model (c) to the data is significantly better than the original modelling; moreover, it reproduces the asymmetry of the signal. Model (a) yields a $K_{m}$ value two times higher than that of model (c). This error is small, considering the very high $\mu / m \approx 20$ ratio. This results from the fortuitous compensation of the natural propensity of model (a) to overestimate $K_{m}$ values with the two-fold error in the time constant for the decrease of CO.

Parkin et al obtained an estimate of $K_{m}$ by averaging over 13 experiments. The value of $\mu / m \approx 20$ that we determined from the experimental data in figure 5 suggest that the authors may have observed some variability in the fitted values of $K_{m}$, which would have been much smaller had model (c) been used.

To ease the modelling of experimental data, we propose in the Experimental section a simple method using the data analysis program QSoas[26] to analyze data.

\section{Conclusion}

We have analysed the responses in current of CODH-modified electrodes to transient exposures to $\mathrm{CO}$ to determine the $K_{m}$ value of the enzyme. We show that failing to take into account mass transport yields to unreliable (generally overestimated) values of $K_{m}$, whereas using the Koutecky-Levich approximation yields to unreliable, underestimated values of $K_{m}$. We derived an equation taking into account the mass transport of substrate, and show that it models perfectly the data, yielding meaningful values of the $K_{m}$ and of the transport parameters.

\section{Acknowledgements}

The authors acknowledge support from CNRS, Agence Nationale de la Recherche (ANR-12-BS08-0014, ANR-14-CE050010) and the A*MIDEX project ( ${ }^{\circ}$ ANR-11-IDEX-0001-02) funded by the «Investissements d'Avenir» French Government program, managed by the French National Research Agency (ANR). The authors are members of the French Bioinorganic Chemistry group (http: //frenchbic.cnrs.fr).

\section{References}

[1] F. A. Armstrong, H. A. Heering, J. Hirst, Reactions of complex metalloproteins studied by protein-film voltammetry, Chem. Soc. Rev. 26 (1997) 169-179. doi:10.1039/CS9972600169.

[2] C. Léger, P. Bertrand, Direct electrochemistry of redox enzymes as a tool for mechanistic studies, Chem. Rev. 108 (7) (2008) 2379-2438. doi: 10.1021/cr0680742.

[3] V. Fourmond, C. Léger, Protein electrochemistry: Questions and answers., Adv. Biochem. Eng. Biotechnol. (advanced online publication) (2016) 1-41. doi:10.1007/10_2015_5016. 
[4] V. Fourmond, C. Greco, K. Sybirna, C. Baffert, P.-H. Wang, P. Ezanno, M. Montefiori, M. Bruschi, I. Meynial-Salles, P. Soucaille, J. Blumberger, H. Bottin, L. de Gioia, C. Léger, The oxidative inactivation of FeFe hydrogenase reveals the flexibility of the H-cluster., Nat. Chem. 6 (4) (2014) 336-342. doi:10.1038/nchem. 1892.

[5] J. G. Jacques, B. Burlat, P. Arnoux, M. Sabaty, B. Guigliarelli, C. Léger, D. Pignol, V. Fourmond, Kinetics of substrate inhibition of periplasmic nitrate reductase, Biochim. Biophys. Acta 1837 (10) (2014) 1801-1809. doi:10.1016/j.bbabio.2014.05.357.

[6] A. Kubas, C. Orain, D. De Sancho, L. Saujet, M. Sensi, C. Gauquelin, I. Meynial-Salles, P. Soucaille, H. Bottin, C. Baffert, V. Fourmond, R. B. Best, J. Blumberger, C. Léger, Mechanism of $\mathrm{O}_{2}$ diffusion and reduction in FeFe hydrogenases., Nat Chem 9 (1) (2017) 88-95. doi:10.1038/ nchem. 2592.

[7] C. F. Megarity, J. Esselborn, S. V. Hexter, F. Wittkamp, U.-P. Apfel, T. Happe, F. A. Armstrong, Electrochemical investigations of the mechanism of assembly of the active-site $\mathrm{H}$-cluster of [FeFe]-hydrogenases, $\mathrm{J}$. Am. Chem. Soc. 138 (46) (2016) 15227-15233. doi:10.1021/jacs $6 \mathrm{~b} 09366$.

[8] S. V. Hexter, F. Grey, T. Happe, V. Climent, F. A. Armstrong, Electrocatalytic mechanism of reversible hydrogen cycling by enzymes and distinctions between the major classes of hydrogenases., Proc. Natl. Acad. Sci. U.S.A. 109 (29) (2012) 11516-11521. doi:10.1073/pnas 1204770109.

[9] V. Fourmond, C. Baffert, K. Sybirna, T. Lautier, A. Abou Hamdan, S. Dementin, P. Soucaille, I. Meynial-Salles, H. Bottin, C. Léger, Steady-state catalytic wave-shapes for 2-electron reversible electrocatalysts and enzymes, J. Am. Chem. Soc. 135 (10) (2013) 3926-3938. doi:10.1021/ ja311607s.

[10] V. Fourmond, C. Léger, Modelling the voltammetry of adsorbed enzymes and molecular catalysts, Current Opinion in Electrochemistry (advanced online publication). doi:10.1016/j.coelec.2016.11.002

[11] H. Pershad, J. Duff, H. Heering, E. Duin, S. Albracht, F. Armstrong, Catalytic electron transport in Chromatium vinosum [NiFe]-hydrogenase: application of voltammetry in detecting redox-active centers and establishing that hydrogen oxidation is very fast even at potentials close to the reversible $\mathrm{H}^{+} / \mathrm{H}_{2}$ value., Biochemistry 38 (28) (1999) 8992-9. doi : 10.1021/bi990108v.

[12] H. A. Heering, J. Hirst, F. A. Armstrong, Interpreting the catalytic voltammetry of electroactive enzymes adsorbed on electrodes, J. Phys. Chem. B 102 (35) (1998) 6889-6902. doi : 10.1021/jp981023r.

[13] T. Reda, J. Hirst, Interpreting the catalytic voltammetry of an adsorbed enzyme by considering substrate mass transfer, enzyme turnover, and interfacial electron transport, J. Phys. Chem. B 110 (3) (2006) 1394-1404. doi:10.1021/jp054783s.

[14] M. Can, F. A. Armstrong, S. W. Ragsdale, Structure, function, and mechanism of the nickel metalloenzymes, CO dehydrogenase, and acetylCoA synthase, Chem. Rev. 114 (8) (2014) 4149-4174. doi:10.1021/ cr400461p.

[15] H. Dobbek, V. Svetlitchnyi, L. Gremer, R. Huber, O. Meyer, Crystal structure of a carbon monoxide dehydrogenase reveals a [Ni-4Fe-5S] cluster., Science 293 (5533) (2001) 1281-1285. doi:10.1126/science. 1061500.

[16] O. Lazarus, T. W. Woolerton, A. Parkin, M. J. Lukey, E. Reisner, J. Seravalli, E. Pierce, S. W. Ragsdale, F. Sargent, F. A. Armstrong, Watergas shift reaction catalyzed by redox enzymes on conducting graphite platelets, J. Am. Chem. Soc. 131 (40) (2009) 14154-14155. doi:10 1021/ja905797w.

[17] A. Parkin, J. Seravalli, K. A. Vincent, S. W. Ragsdale, F. A. Armstrong, Rapid and efficient electrocatalytic $\mathrm{CO}_{2} / \mathrm{CO}$ interconversions by Carboxydothermus hydrogenoformans $\mathrm{CO}$ dehydrogenase I on an electrode, J. Am. Chem. Soc. 129 (34) (2007) 10328-10329. doi:10.1021/ ja073643o.

[18] V. C.-C. Wang, M. Can, E. Pierce, S. W. Ragsdale, F. A. Armstrong, A unified electrocatalytic description of the action of inhibitors of nickel carbon monoxide dehydrogenase, J. Am. Chem. Soc. 135 (6) (2013) 2198-2206. doi:10.1021/ja308493k.

[19] J. Hadj-Saïd, M.-E. Pandelia, C. Léger, V. Fourmond, S. Dementin, The carbon monoxide dehydrogenase from Desulfovibrio vulgaris, Biochim. Biophys. Acta 1847 (12) (2015) 1574-1583. doi:10.1016/j.bbabio. 2015.08.002.
[20] M. Merrouch, J. Hadj-Saïd, L. Domnik, H. Dobbek, C. Léger, S. Dementin, V. Fourmond, $\mathrm{O}_{2}$ inhibition of Ni-containing $\mathrm{CO}$ dehydrogenase is partly reversible, Chem. Eur. J. 21 (52) (2015) 18934-18938. doi:10.1002/chem.201502835.

[21] A. J. Bard, L. R. Faulkner, Electrochemical methods: Fundamentals and Applications, 2nd Edition, Wiley, 2000.

[22] J. Newman, K. E. Thomas-Alyea, Electrochemical Systems, 3rd Edition, Wiley-Interscience, 2004.

[23] C. Léger, S. Dementin, P. Bertrand, M. Rousset, B. Guigliarelli, Inhibition and aerobic inactivation kinetics of Desulfovibrio fructosovorans $\mathrm{NiFe}$ hydrogenase studied by protein film voltammetry., J. Am. Chem. Soc. 126 (38) (2004) 12162-72. doi : 10.1021/ja046548d.

[24] C. Orain, L. Saujet, C. Gauquelin, P. Soucaille, I. Meynial-Salles, C. Baffert, V. Fourmond, H. Bottin, C. Léger, Electrochemical measurements of the kinetics of inhibition of two $\mathrm{FeFe}$ hydrogenases by $\mathrm{O}_{2}$ demonstrate that the reaction is partly reversible, J. Am. Chem. Soc. 137 (39) (2015) 12580-12587. doi:10.1021/jacs.5b06934.

[25] D. Wise, G. Houghton, Diffusion coefficients of neon, krypton, xenon, carbon monoxide and nitric oxide in water at $10-60^{\circ} \mathrm{C}$, Chem. Eng. Sci. 23 (10) (1968) 1211-1216. doi:10.1016/0009-2509 (68)89029-3.

[26] V. Fourmond, QSoas: a versatile software for data analysis, Anal. Chem. 88 (10) (2016) 5050-5052. doi:10.1021/acs . analchem. 6b00224. 